A. Weisbecker, M. Burmester \& A. Schmidt (Hrsg.): Mensch und Computer 2015 Workshopband, Stuttgart: Oldenbourg Wissenschaftsverlag, 2015, S. 405-410.

\title{
HaptOSM - Creating tactile maps for the blind and visually impaired
}

\author{
Daniel Hänßgen \\ University of Applied Sciences and Arts Hanover \\ Faculty IV - Applied Computer Sciences / Faculty II - Mechanical Engineering
}

\begin{abstract}
This paper describes the latest accomplishments on the current research that is based on the master's thesis "Ein System zur Erstellung taktiler Karten für blinde und sehbehinderte Menschen" (German for "A system creating tactile maps for blind and visually impaired people") (Hänßgen, 2012). The system consists of two parts. The first part is new software especially designed and developed for creating tactile maps addressing the needs of blind and visually impaired people on tactile information. The second is an embossing device based on a modified CNC (computer numerical control) router. By using OpenStreetMap-data, the developed system is capable of embossing tactile maps into Braille paper and writing film.
\end{abstract}

\section{Motivation}

Being able to move freely in public spaces should be a matter of course for everybody in today's society. Nevertheless, there is little opportunity, especially for the blind, to orientate themselves in advance before visiting an unknown place. Of course there are already commercial tactile maps for blind and visually impaired people available today. The most common technique for manufacturing tactile maps is called thermoforming (Perkins, 2002). But due to high production costs and long manufacturing time, those maps are limited to very few places and are rarely recreated. The map of Marburg, a city with many blind residents, is a possible example. Although a new edition was published in early 2015, 30 years elapsed between this edition and the previous one. Then, as now, it costs $€ 99$ measuring 40 by 40 centimetres (Stehlik, 2008). This price still is only possible with subsides and a fairly high number of copies. Manufacturing a single copy of an arbitrary chosen region would cost at least $€ 2500$ (personal correspondence with the DZB http://www.dzb.de). During early research on this topic a high demand on inexpensive highly up-to-date tactile maps was stated by the blind community. HaptOSM project has the ambition to facilitate orientation in public spaces and buildings for blind and visually impaired people by creating tactile maps on Braille paper 
writing film. Due to an almost entirely automated manufacturing process, these maps are affordable with a single copy.

\section{Idea}

First of all, while creating maps, the data source plays an important role. The data needs to be affordable and also should contain valuable information for the blind and visually impaired. Fortunately, map data meeting these requirements is available by the OpenStreetMap-Project (http://www.openstreetmap.org). Further details on OpenStreetMap-data for blind and visually impaired people can be found at the OpenStreetMap-wikipage "OSM for the blind" (http://wiki.openstreetmap.org/wiki/OSM_for_the_blind). Covering almost all parts of the world, and being updated every minute, OpenStreetMap provides data licenced with the OpenDatabase-License (ODbl) (http://opendatacommons.org/licenses/odbl/1.0/). The license allows the usage of the data even for commercial purposes. OpenStreetMap data includes both traditional information about roads and frontiers as well as information especially for the blind and visually impaired, like tactile pavement surfaces, pedestrian crossings and pedestrian sound signals. The basic idea of HaptOSM project is to enable easy access for blind and visually impaired people to the free map data of OpenStreetMap.

A new method embossing braille paper and writing film has been developed. Individual tactile maps can be easily manufactured on demand with almost no human interaction needed for manufacturing. The concept includes the development and construction of the necessary hardware and in particular, the implementation of the necessary software, preparing the OpenStreetMap-data for embossing. Close details on software and hardware are stated in the following chapters.

\section{Research and development}

\subsection{Hardware}

Instead of exploring entirely new ways regarding the material out of which the maps are manufactured, it was decided to use Braille paper and writing film. The ability to understand tactile information depends on the user's experience, exploration strategies, training and skills (Thinus-Blanc and Gaunet, 1997). Therefore, stated materials were chosen since they are familiar to most blind people. The Braille paper being used in this project is the same paper that blind books are made of. The writing film is about $0.12 \mathrm{~mm}$ thick and made of white plastic. It is tear- and waterproof and therefore, lasts considerably longer than paper. In addition these two features make the film easier to emboss. The left side of figure 1 shows the 
working prototype of the embossing machine already in use for production of tactile maps at https://haptosm.de .

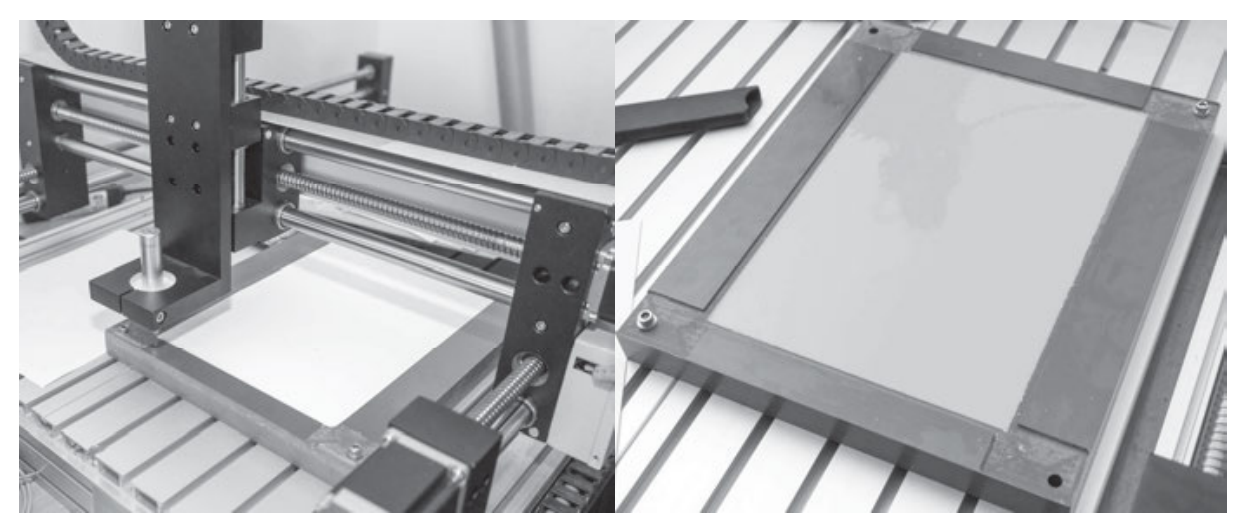

Figure 1: Prototype embossing machine and holding device

Essentially, it is a modified CNC router. Instead of the milling motor the machine has an embossing pen mounted onto the portal. The machine provides three degrees of freedom (translation in $\mathrm{X}, \mathrm{Y}, \mathrm{Z}$ direction) allowing the pen to emboss arbitrary lines and dots onto a sheet of paper or film. To secure the sheet on the machine and prevent it from moving, a special holding device was designed. The right side of figure 1 shows a close up of the device. Two areas with different depths and sizes were milled out of the plastics. The larger area has exactly the size of a DIN A4 sheet, preventing the sheet from slipping. The smaller area is about $4 \mathrm{~mm}$ deep and was filled out with a soft silicone, allowing the pen to emboss paper or film. The machine is working with G-Code as input. G-Code - in short - is a list of points that the machine is supposed to approach. The machine itself is controlled by LinuxCNC, an OpenSource-Software running on a special Linux designed for real-time applications (http://www.linuxcnc.org). The pen is simply lowered into the paper and then moved to emboss. This allows speeds of $600 \mathrm{~mm}$ per minute using Braille paper and $1400 \mathrm{~mm}$ per minute using writing film. On average, 10-15 minutes are required to complete a map.

\subsection{Software}

In addition to the hardware, software was developed that translates the OpenStreetMap-data into G-Code. The software called OSMtoCNC is written in Java and kept as modular as possible allowing easy adjustments for other data sources. OSMtoCNC takes several parameters to customize the resulting map for the user's needs. Essential parameters like location, scale and language are mandatory. Other parameters like how different kinds of streets should be represented can also be chosen to accommodate to the wishes and needs of the user. OSMtoCNC calculates the area needed upon the given parameters and accesses the OpenStreetMap-data via Overpass API (http://overpass-api.de). An Open-Source-Software 
with powerful query language allowing very detailed selection of the map data. This data is then processed through several stages. Unfortunately, showing all stages would massively exceed the scope of this paper. The software has been thoroughly tested and is functional within desired parameters. OSMtoCNC makes use of parallel programming allowing short processing times even on multipage maps, since the pages are processed in separate threads.

\subsection{Tactile elements of the map}

The map was designed following the principle "Keep it simple" (Edman, 1992) and always asking the question "Is this information necessary?" (Edman, 1992). As a result, information added onto the map has to be a surplus. Bearing in mind that the resolution of the fingers are significantly lower than that of the eyes (Schiff and Foulke, 1982), only a limited amount of different symbols are used to ensure distinguishable information without confusing the user.

The tactile elements used were carefully developed in cooperation with blind personnel at the "Blinden- und Sehbehindertenverband Niedersachsen e. V. (BVN)" (Association of the Blind and Visually Impaired Lower Saxony) (http://blindenverband.org). They also tested the first maps. The results can be found in "Tests and conclusions".

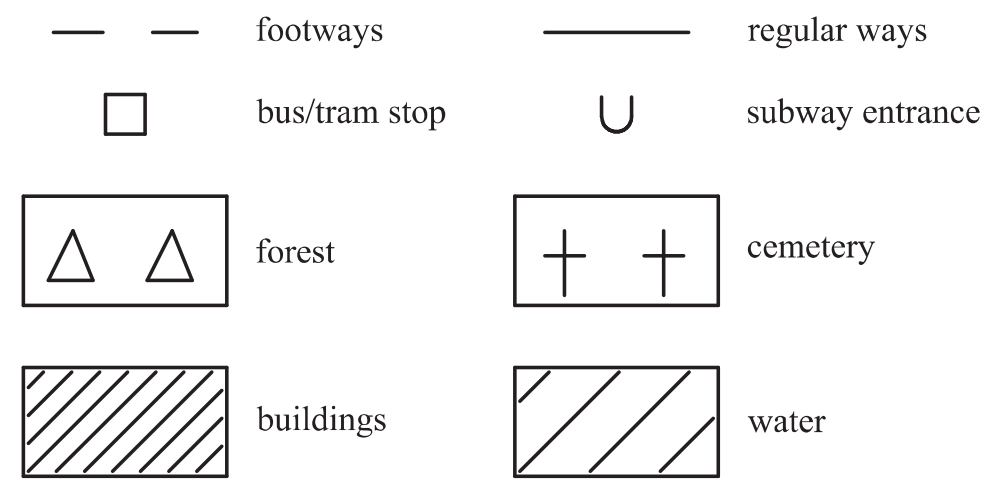

Figure 2: Tactile elements

Figure 2 shows a schematic representation of the tactile elements which are described in detail below.

Footways are rendered as dashed lines, whereas all other streets and ways are rendered as continuous lines. This differentiation might seem very simple, but since blind people are most often traveling by foot, this was stated by the BVN staff to be enough information. Of course OpenStreetMap offers a wide range of different way types. The representation of the most common types (twelve and counting) can easily be changed via configuration file, addressing special needs.

Since the machine is using an embossing pen, areas are not possible to emboss in Braille paper. The paper would tear. Therefore areas are rendered as repeating symbols. For example: A 
forest is rendered as triangles pointing upwards. These are placed in a repeating pattern allowing easy recognition. An outline represents the border. This was necessary because cutting of the triangle pattern at the actual forest's border would create unknown "symbols" that would easily irritate the user. The same method is used for cemetery rendered using crosses instead of triangles.

Besides going by foot the local public transport is an important way of transportation for blind and visually impaired people. Therefore, subway station entries and bus/tram stops are displayed on the maps. Subway station entries are rendered as capital "U" whereas bus/tram stops are rendered as small squares. Of course these symbols are explained on an extra sheet or directly on the website https://haptosm.de.

Latest implementations into the OSMtoCNC software are buildings as well as rivers and lakes. The challenge is similar to the symbols for the forest and the cemetery because water is usually mapped as area. Buildings are rendered as an outline filled with close hatching. The distance between two lines defaults to $1 \mathrm{~mm}$. Water is rendered as outline filled with wide hatching, defaulting to $3 \mathrm{~mm}$ distance between two lines. The lines are aligned at a 45 degree angle. Of course the distance between two lines and the angle are configurable.

Still in development are pedestrian crossings. The OpenStreetMap allows different kinds of mapping styles for pedestrian crossings. Finding the right unique symbols representing different kinds of crossings is still in research. For example small pedestrian crossings are just a node on a way, whereas pedestrian crossings over larger intersections are often mapped as footways across the streets. The latter are already shown on the maps of this project but unfortunately tend to confuse the user, because the information density is very high on such intersections.

\section{Tests and conclusion}

At the time of writing this paper, an empirical study is being planned and executed. The results of this study are going to be presented at the workshop in September. Nevertheless, tests with individuals have been made which have shown very promising results. Working close with the people at BVN, blind specialists took the map to the test. In their opinion the map has a great readability and the Braille script feels like in books for the blind.

Addressing visually impaired people is yet a work in progress. At the University of Applied Sciences and Arts Hanover a bachelor's thesis is being written, developing a plug-in for OSMtoCNC. This plug-in will then allow creating a PDF file containing basic colours representing certain areas (e.g. water being blue and forest being green). This will allow visually impaired people to use their remaining eyesight in addition to the tactile information.

HaptOSM technology is currently functional in a public beta stage and maps can already be ordered via https://haptosm.de. 


\section{Bibliography}

Edman, Polly K. (1992). Tactile Graphic. American Foundation for the Blind, 197.

Hänßgen, D. (2012). Ein System zur Erstellung taktiler Karten für blinde und sehbehinderte Menschen. Hochschule Hannover - Fakultät IV - Angewandte Informatik.

Perkins, C. (2002). Cartography: progress in tactile mapping. Prog. Hum. Geogr. 24 (4), 521 - 530.

Schiff, W., Foulke, E. (1982). Tactual Perception: a Sourcebook. Cambridge University, Pr.7

Stehlik, Uwe (2008). Taktile Medien - Orientierungs- und Informationssysteme. Marburg: Deutsche Blindenstudienanstalt e.V.

Thinus-Blanc, C., Gaunet, F. (1997). Representation of space in blind persons: vision as a spatial sense? Psychological Bulletin, 121, 20 - 42.

\section{Contact information}

Daniel Hänßgen

Hochschule Hannover - University of Applied Sciences and Arts Hanover

Fakultät II - Maschinenbau

Bismarckstraße 2

30173 Hannover

Mobile: 0176-61084118

E-mail: daniel.haenssgen@hs-hannover.de

Website: https://haptosm.de 